Background and aims Dectin-1 is a c-type lectin like receptor that signals via syk and is involved in anti-fungal immunity. Dectin-1 was found to trigger experimental inflammatory arthritis, and likely play a role in the pathogenesis of some autoimmune diseases. This study aimed to examine dectin-1 expression and function of circulating CD14+ monocytes and monocyte-derived dendritic cells (MDDCs) in patients with systemic lupus erythematosus (SLE)

Methods SLE patients with active and inactive diseases and healthy subjects were recruited. Dectin-1 agonists including curdlan, zymosan and toll-like receptor agonists Pam3CSK4 (TLR2) and LPS (TLR4) were used to stimulate monocytes and/or MDDCs. Dectin-1, ROS and phosphorylated-syk (pSyk) were measured by flow cytometry. Cytokine profile was measured by and multi-bead immunoassay.

Results Dectin-1 expressing monocytes was significantly lower in active SLE patients compared to inactive patients and healthy controls. The absolute count of dectin-1 expressing monocytes correlated significantly and inversely with SLEDAI, anti-dsDNA antibody level and C4. Despite this, ROS production upon stimulation by dectin-1 agonists was comparable. Stimulation of dectin-1 led to activation and maturation of MDDCs. SLE MDDCs showed higher p-Syk activation compared to normal MDDCs upon dectin-1 stimulation. Curdlanstimulated MDDCs produced higher levels of IL-1 $\beta$, IL-23 and TNF- $\alpha$. Adding TLR2 agonist to curdlan, SLE MDDCs produced significantly higher level of IL-1 $\beta$ compared to normal MDDCs.

Conclusions Active SLE patients had significantly lower circulating dectin-1 expressing monocytes which produced comparable level of ROS compared to inactive patients and healthy subjects. Dectin-1 agonists led to significantly higher Th17 promoting cytokines upon co-stimulation with TLR2 in SLE MDDCs.

\section{TMEM173/STING IS CRUCIAL FOR LUPUS DEVELOPMENT IN FCGR2B-DEFICIENCY MICE}

${ }^{1}$ P Pisitkun* ${ }^{2} \mathrm{~A}$ Thim-Uam, ${ }^{3} \mathrm{M}$ Tansakul, ${ }^{2} \mathrm{~B}$ Wongprom, ${ }^{4} \mathrm{~A}$ Leelahavanichkul, ${ }^{5} \mathrm{~S}$ Paludan, ${ }^{2}$ T Pisitkun. ${ }^{1}$ Faculty of Medicine Ramathibodi Hospital, Department of Medicine- Division of Allergy- Immunology- and Rheumatology, Bangkok, Thailand; ${ }^{2}$ Faculty of MedicineChulalongkorn University, Chulalongkorn University Systems Biology Centre, Bangkok, Thailand; ${ }^{3}$ Faculty of Medicine Ramathibodi Hospital, Translational Medicine Program, Bangkok, Thailand; ${ }^{4}$ Faculty of Medicine- Chulalongkorn University, Department of Microbiology, Bangkok, Thailand; ${ }^{5}$ Aarhus University, Department of Biomedicine, Aarhus, Denmark

\subsection{6/lupus-2017-000215.349}

Background and aims Type I interferon is one of the most critical cytokines involving in lupus pathogenesis. The activation of endosomal nucleic acid sensors leading to type I IFN production empowers the lupus phenotypes in several mouse models. The signalling of cytosolic DNA sensor also induces type I IFN production and the role in lupus disease are not clear. Stimulator of interferon genes (Sting), also known as transmembrane protein 173 (TMEM173) and MPYS/MITA/ ERIS, is a cytosolic DNA sensor which recognised cyclic diGMP and subsequently stimulated type I interferon production. The Fcgr2b-deficient mice develop spontaneous lupus phenotypes which are splenomegaly, the presence of antinuclear antibodies (ANA) and fatal glomerulonephritis. The polymorphisms of FCGR2B associates with the increases of lupus susceptibility in human. The goal of the study is to identify the role of Sting in lupus mouse model.

Methods The Sting-deficient mice were bred with the Fcgr2bdeficient mice to create double deficient mice and control littermates. The mice were analysed for survival rate, autoantibodies production, severity of pathology, gene expression profiles, and immunophenotypes.

Results In the absence of Sting, the Fcgr2b-deficient mice survived longer and the level of ANA and anti-dsDNA antibody considerably reduced. The glomerulonephritis in the doubledeficient mice also ameliorated. The expression of interferon

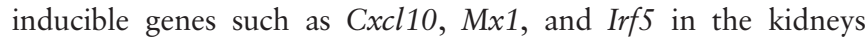
of the double-deficient mice was significantly lower than the Fcgr2b-deficient mice.

Conclusions Sting-mediated signalling pathway plays the substantial role in lupus pathogenesis of the $F \operatorname{cg} r 2 b$-deficient mice. Blocking Sting function may be the advantage for treatment in lupus patients.

\section{TOLL-LIKE RECEPTOR 7 SIGNALLING DRIVES TRANSITIONAL B CELLS EXPANSION AND AUTOANTIBODY PRODUCTION IN SYSTEMIC LUPUS ERYTHEMATOSUS}

${ }^{1} \mathrm{~T}$ Wang ${ }^{*},{ }^{2} \mathrm{~J}$ Marken, ${ }^{3} \mathrm{~K}$ Cerosaletti, ${ }^{1} \mathrm{M}$ Li, ${ }^{2} \mathrm{~K}$ Elkon, ${ }^{1} \mathrm{X}$ Zeng, ${ }^{2} \mathrm{~N}$ Giltiay. ${ }^{1}$ Peking Union Medical College Hospital, Rheumatology, Beijing, China; '2University of Washington, Rheumatology, Seattle, USA; ${ }^{3}$ Benaroya Research Institute at Virginia Mason, Translational Research Program, Seattle, USA

\subsection{6/lupus-2017-000215.350}

Background and aims Toll-like receptor 7 (TLR7) has been implicated in B cells activation and the generation of pathogenic autoantibodies. Newly-formed transitional (TR) B cells are enriched in autoreactive specificities and are increased in some SLE patients. This study was undertaken to examine a possible link between the TR B cells expansion/activation and TLR7 levels in SLE.

Methods PBMCs were collected from SLE patients and healthy donors and analysed for the expression of TLR7, TLR9 and IFN-responsive genes by RT-PCR. The frequencies of B cell populations were analysed by flow cytometry. BAFF titers were analysed by ELISA. TLR7 variant rs3853839(C/G) was detected by Taqman 5'-allele discrimination assay. TR B cells were primed with IFN $\alpha$ and stimulated with TLR7 ligands in vitro.

Results High expression levels of TLR7 in SLE patients positively correlated with IFN signature and disease activity, but not with BAFF titers. SLE patients with high levels of TLR7 (TLR7 ${ }^{\text {hi }}$ group) showed an expansion of $\mathrm{CD} 19^{+} \mathrm{CD} 38^{\text {high }} \mathrm{CD} 24^{\text {high }} \mathrm{CD} 10^{+}$TR B cells. Overall, frequencies of TR B cells positively correlated with the levels of TLR7, but not TLR9. SLE patients, carrying a risk G allele, had increased TLR7 expression and TR cell frequencies, compared to non-risk allele carriers. TLR $7^{\text {hi }}$ SLE patients showed increased autoantibody titers and skewing towards Sm/RNP antigens. Upon IFN $\alpha$ priming, TR B cells up-regulated TLR7 and differentiated into plasmablasts in response to TLR7ligand stimulation.

Conclusions Our findings suggest that dysregulation of TLR7 in SLE might drive the expansion and promote the activation of TR B cells, which might be a source of autoantibodies. 


\section{AUTOIMMUNE-ASSOCIATED GENE CLEC16A REGULATES NLRP3 BUT NOT AIM2 INFLAMMASOME PATHWAY IN HUMAN MACROPHAGES}

WMM Li, ALY Yim*, VSF Chan, CS Lau. The University of Hong Kong, Department of Medicine - Division of Rheumatology and Clinical Immunology, Hong Kong, Hong Kong S. A.R

\subsection{6/lupus-2017-000215.351}

Background and aims C-type lectin domain family 16 member A (CLEC16A) has been associated with autoimmune diseases such as systemic lupus erythematosus, multiple sclerosis and type I diabetes in various genome-wide association studies. Subsequent studies revealed that mouse/human CLEC16A and its Drosophila homolog endosomal maturation defective isoform A (EMA) are involved in different aspects of autophagy, the regulated degradation of cellular components that are in excess or dysfunctional. Crosstalk between autophagy and inflammasome activity of innate immune responses has been reported and inflammasomes are activated in various autoimmune diseases. We thus sought to investigate the role of CLEC16A in inflammasome pathway in this study.

Methods Functional genetic studies of CLEC16A in NLRP3 and AIM2 inflammasome pathways using monocyte-derived macrophages isolated from peripheral blood mononuclear cells of healthy individuals were performed.

Results During induction of NLRP3 inflammasome pathway by nigericin, a knockdown of CLEC16A using specific siRNAs inhibited secretion of interleukin-1 $\beta$ (IL-1 $\beta$ ), an inflammasome pathway effector. Its secretion during AIM2 inflammasome induction by intracellular dsDNA poly(dA:dT) however was not affected in the siCLEC16A group. The induction of NLRP3 mRNA level upon lipopolysaccharide stimulation was suppressed in the siCLEC16A group. No significant changes in mRNA levels was observed in other selected genes of NLRP3 inflammasome pathway, namely the adaptor protein ASC, interleukin-1 converting enzyme caspase- 1 and precursor proIL-1 $\beta$.

Conclusions These data suggest that CLEC16A regulates NLRP3 but not AIM2 inflammasome pathway and affects IL$1 \beta$ secretion in part via NLRP3 level. The mechanism involved and its association with autoimmune diseases such as systemic lupus erythematosus remains to be elucidated.

\section{FROM ANIMAL MODELS TO HUMAN - A PHENOTYPIC AND FUNCTIONAL STUDY OF PDCS}

'ZZ Zhou, ${ }^{1} \mathrm{M}$ Jianyang, ${ }^{2} \mathrm{Q}$ Rong, ${ }^{1} \mathrm{~S}$ Nan*. ${ }^{1}$ Renji Hospital- School of Medicine- Shanghai Jiaotong University, Shanghai Institute of Rheumatology, Shanghai, China; ${ }^{2}$ Shanghai Institutes for Biological Sciences SIBS and Shanghai Jiao Tong University School of Medicine SJTUSM- Chinese Academy of Sciences CAS, Institute of Health Sciences, Shanghai, China

\subsection{6/lupus-2017-000215.352}

Background and aims To study the role of plasmacytoid dendritic cells(pDCs) in the pathogenesis of systemic lupus erythematosus(SLE)

Methods Totally 9 mouse strains were studied including NZB, NZW, NZBW F1, MRL/lpr, MRL/Mp, BXSB/Mp, BXSB.B6. Yaa, B6.SLE1.2.3 and C57BL/6. Spleen, thymus, bone marrow and lymph node pDCs were collected from mice in different disease stages by using Nycodenz enrichment and sorting systems. Human pDCs from healthy donor and SLE patients were isolated by using BDCA4 beads selection. Mouse pDCs were stimulated with ODN2216 and Poly U for Tlr9 and Tlr7 respectively. Human pDCs were stimulated with ODN2216 and R837 for Tlr9 and Tlr7 respectively. After 18 hour for human and 36 hour for mouse, supernatant was collected for ELISA test. IFNa, TNFa, and IL6 were tested.

Results Bone marrow pDC could produce much higher IFNa than pDCs from spleen, thymus and lymph node in all tested strains. pDCs from NZB, NZBW F1 could produce higher IFNa than those from other strains. Spleen pDCs from MRL/ lpr and MRL/Mp mice could produce higher levels of IFNa via Tlr7 than Tlr9 stimulation. All lupus-prone mice except $\mathrm{BXSB} / \mathrm{Mp}$ and B6.SLE1.2.3 strains have higher total pDCs numbers. Cytokine-producing ability of pDCs were reduced in mice with advanced disease stage. pDCs from SLE patients could produce higher IFNa than those from a healthy donor. Conclusions Phenotype and function of pDCs were largely dependent on their genetic background. Activation and function alterations of pDCs were observed in a lupus model. Hyperactive of pDC from SLE patients may contribute to lupus pathogenesis.

\section{Microbiome, infections, probiotics and nutritional factors in autoimmunity}

\section{COFFEE CONSUMPTION AND CLINICAL OUTCOMES IN COLOMBIAN PATIENTS WITH SYSTEMIC LUPUS ERYTHEMATOSUS}

MA Alzate ${ }^{* 1} 1^{2} \mathrm{~F}$ Ochoa, ${ }^{1} \mathrm{P}$ Ortiz-Salazar, ${ }^{1} \mathrm{D}$ Hernandez-Parra, ${ }^{1} \mathrm{R}$ Pineda. ${ }^{1}$ ARTMEDICA, Clinical information group, Medellin, Colombia; ${ }^{2}$ Universidad CES, Public Health Group, Medellin, Colombia

\subsection{6/lupus-2017-000215.353}

Background and aims This study was performed to analyse the influence of coffee consumption on clinical outcomes in colombian patients with systemic lupus erythematosus (SLE). Methods A cross-sectional analytical study was conducted in 731 Colombian patients with SLE in whom coffee consumption and its frequency was assessed. Differences in clinical outcomes (i.e., cardiovascular disease, age-at-onset, organ damage, polyautoimmunity, acute activity determined by SLEDAI, and clinical remission) were determined between drinkers and nondrinkers, as well as by frequency of consumption. Association was examined by chi-square and multivariate regression analyses.

Results Sociodemographic and clinical characteristics of the patients are shown in table 1 . Out of a total of 731 patients, $70 \%$ were current coffee drinkers, $57 \%$ reported daily consumption, $10 \%$ weekly consumption and $3 \%$ monthly consumption. Coffee intake was found to be associated with SLE age at onset, cardiovascular disease and 6 months clinical remission (Table 2). According to the frequency of intake, daily consumption was associated to reduced risk of early ageat-onset and a positive association with 6 months clinical remission (OR: 0,45 95\% CI $0,25-0,81$ and OR: 1,55 95\% CI 1,07-2,25, respectively). No differences were found for organ damage, polyautoimmunity and acute SLE activity. Conclusions In Colombian SLE patients with coffee consumption, a reduced risk of early age-at-onset and cardiovascular disease was recognised. Also, a positive association with 6 month clinical remission was found. Due to the 